\title{
Noise-induced signal corruption in nonlinear Fourier-based optical transmission system in the presence of discrete eigenvalues
}

\author{
Maryna L. Pankratova ${ }^{1}{ }^{a}$, Anastasiia Vasylchenkova ${ }^{1}$, Jaroslaw E. Prilepsky ${ }^{1}{ }^{\mathrm{b}}$ \\ ${ }^{1}$ Aston Institute of Photonic Technologies, Aston University, Aston Triangle, B4 7ET, Birmingham, UK \\ pankratm@aston.ac.uk
}

Keywords: Optical Communications, Nonlinear Fourier Transform, Noise, Soliton, Correlation Properties

Abstract: We present the numerical analysis of the correlation properties of the amplifier spontaneous emission (ASE) noise transformed into the nonlinear Fourier (NF) domain, addressing the noise-induced corruptions in the communication systems employing the nonlinear Fourier transform (NFT) based signal processing. In our current work we deal with the orthogonal frequency division multiplexing (OFDM) modulation of a continuous NF spectrum and account for the presence of discrete (soliton) eigenvalues. This approach is aimed at extending our previous studies that referred to the modulation of continuous NF spectrum only. The effective noise covariance functions are obtained from numerical simulations for a range of propagation distances, values of discrete eigenvalue, and different effective signal power levels. We report the existence of the correlations between the continuous and discrete parts of the NF spectrum.

\section{Introduction}

The rise of interest to the nonlinear frequency division multiplexing technique (NFDM), the optical transmission method based on the NFT signal processing and the modulation of effective modes inside the NF domain, can be explained by effective absence of the nonlinear cross-talk between the different multiplexed signal components provided by this method (Turitsyn et al., 2017; Yousefi and Kschischang, 2014; Le et al., 2017). In turn, the latter is believed to the major source of the capacity degradation in modern optical transmission lines (Essiambre et al., 2010). For the NFDM, the data transmission takes place inside the NF domain, where the residual weak nonlinear cross-talk occurs only because of the deviation of the true optical channel from the idealised mathematical model. At the same time, the invevitable presence of the optical noise in the channel, occuring due to the amplification, can significantly hamper the quality and reach of the NFT-based transmission, affecting both continous and discrete parts of the NF (Civelli et al., 2017; Garcia-Gomez and Aref, 2019). Therefore, the study of the noise-induced signal corruption occurring inside the NF domain constitutes an important direction in the research related to

\footnotetext{
a (iD) https://orcid.org/0000-0002-5974-6160

b (i) https://orcid.org/0000-0002-3035-4112
}

improvement of the NFT-based optical transmission system's throughput.

The NFT-based optical transmission involves three basic steps (Turitsyn et al., 2017): (i) mapping the signal from the NF spectral domain to the spacetime domain using the inverse NFT, INFT; (ii) the signal propagation inside the NFT domain that actually boils down to the phase rotation of individual NF spectral components obeying the linear dispersion law; (iii) the NFT signal processing at the receiver and compensation of the accumulated phase rotation. However, as noted before, in practice, the real fibre differs from the idealised mathematical model attributed to the NFT operations. The main master model governing the light propagation in a idealised lossless single mode fibre is the lossless nonlinear Schrödinger equation (NLSE). The NLSE for the envelope electric field function $q(z, t)$ in presence of ASE noise can be written down as

$$
i q_{z}-q_{t t}-2|q|^{2} q=\eta(z, t),
$$

where $z$ and $t$ are the normalised distance along the fibre and the normalised retarded time in the frame co-moving with the envelope, respectively (Agrawal, 2012). The presence of ASE is taken into account via the $\eta(z, t)$ term in the right hand side of the equation, modelled as the distributed additive white Gaussian noise (AWGN) with zero mean and normalised power 
spectral density $D$ :

$$
\mathbb{E}\left[\eta(z, t) \eta^{*}\left(z^{\prime}, t^{\prime}\right)\right]=2 D \delta\left(t-t^{\prime}\right) \delta\left(z-z^{\prime}\right),
$$

where $\mathbb{E}[\ldots]$ stands for expectation value. Eq. (1) assumes ideal distributed Raman amplification with full compensation of the fibre loss. However, this modes can also serve as a good leading approximation for the path-averaged description of the light propagation in the presence of lumped amplification (Le et al., 2015; Kamalian et al., 2017).

The NLSE, Eq. (1), with the zero right hand side is integrable, meaning that the explicit forms of the forward and inverse NFT operation attributed to such an equation are known (Zakharov and Shabat, 1972). The forward NFT of a given localised (having a finite norm) signal can be calculated by considering the Zakharov-Shabat problem for two auxiliary functions $v_{1}(t, \zeta)$ and $v_{2}(t, \zeta)$ :

$$
\begin{gathered}
\frac{\partial v_{1}(t, \zeta)}{\partial t}=q(t) v_{2}-i \zeta v_{1}, \\
\frac{\partial v_{2}(t, \zeta)}{\partial t}=-\bar{q}(t) v_{1}+i \zeta v_{2},
\end{gathered}
$$

In Eq. (2) the input pulse shape $q(t)$ (we drop the dependence on $z$ ) acts as an effective potential and the overbar here and below means the complex conjugate. The spectral parameter $\zeta$ entering Eq. (2) is a generally complex quantity, $\zeta=\xi+i \rho$, playing the role of the nonlinear analogue of conventional frequency. The "potential" $q(t)$ decays as $t \rightarrow \pm \infty$. To define the NF spectral data, one fixes two linearly-independent left Jost solutions of Eq. (2), $\phi(t, \zeta)=\left[\phi_{1}, \phi_{2}\right]^{T}$ with the initial condition at the left infinity, and two right Jost functions, $\psi(t, \zeta)=\left[\phi_{1}, \phi_{2}\right]^{T}$, fixed by the condition at the right infinity:

$$
\begin{gathered}
\phi(\zeta, t) \sim\left(\begin{array}{l}
1 \\
0
\end{array}\right) e^{-i \zeta t,} \quad t \rightarrow-\infty, \\
\psi(\zeta, t) \sim\left(\begin{array}{l}
0 \\
1
\end{array}\right) e^{i \zeta t}, \quad t \rightarrow \infty .
\end{gathered}
$$

The right and left Jost functions are linearly dependent and relate through the Jost scattering coefficients $a(\zeta)$ and $b(\zeta)$; these two complex quantities constitute the essecne of the NFT signal decomposition. In the explicitly form, these scattering coefficients for the real spectral parameter $\zeta=\xi$ can be obtained as

$$
a(\zeta)=\lim _{t \rightarrow \infty} \phi_{1}(t, \zeta) e^{i \zeta t}, \quad b(\zeta)=\lim _{t \rightarrow \infty} \phi_{2}(t, \zeta) e^{-i \zeta t}
$$

This definition is typically used in the numerical computation of the NFT spectrum (Vasylchenkova et al., 2019). For the right reflection coefficient, $r(\xi)$, which gives us the continuous part of the NF spectrum, we have the following expression:

$$
r(\zeta)=b(\zeta) / a(\zeta)
$$

The unperturbed $z$-evolution of the Jost coefficients $a(\zeta), b(\zeta)$, and right reflection coefficient is given by

$$
\begin{aligned}
& \frac{\partial a}{\partial z}=0, \quad \frac{\partial b}{\partial z}=2 i \zeta^{2} b(\zeta), \\
& \frac{\partial r}{\partial z}=2 i \zeta^{2} r(\zeta) .
\end{aligned}
$$

In addition, the NF decomposition of a given potential can include discrete spectrum component: the complex eigenvalues and the associated complex amplitudes. The former are defined as the zeros of the first Jost coefficient $a\left(\zeta_{k}\right)=0, \mathfrak{I}\left[\zeta_{k}\right]>0$, while the latter are the residues of the right coefficient $r(\xi)$ at the given eigenvalue (Vasylchenkova et al., 2019): $C_{k}=b\left(\zeta_{k}\right) / a^{\prime}\left(\zeta_{k}\right)$. Their unperturbed dynamics are also trivial and follows from Eqs. (6):

$$
\begin{aligned}
& \frac{\partial \zeta_{k}}{\partial z}=0, \\
& \frac{\partial C_{k}}{\partial z}=2 i \zeta_{k}^{2} C_{k} . .
\end{aligned}
$$

In the presence of the deviation from the noiseless NLSE, the dynamics of NF spectrum quantities, Eqs. (6), (7), changes. Applying the perturbation theory to the to the evolution of nonlinear spectral data (Derevyanko et al., 2016; Kaup, 1976; Kaup and Newell, 1978), the perturbed $z$-evolution of the reflection coefficient change in the leading order with respect to the perturbation is given by the following equations (cf. Eqs. (6)-(7)):

$$
\begin{aligned}
& \frac{\partial r}{\partial z}-2 i \xi^{2} r=-\frac{1}{a^{2}} I[\phi, \phi], \\
& \frac{\partial \zeta_{k}}{\partial z}=-\left.C_{k} I[\psi, \psi]\right|_{\zeta_{k}}
\end{aligned}
$$

where $I[u, v]$ is the projection of the noisy perturbation $\eta(t, z)$ on the corresponding unperturbed squared Jost functions:

$$
\begin{array}{r}
I[u, v ; \zeta]=\int_{-\infty}^{\infty} d t\left[\eta(t, z) u_{2}(\zeta, t, z) v_{2}(\zeta, t, z)\right. \\
\left.+\bar{\eta}(t, z) u_{1}(\zeta, t, z) v_{1}(\zeta, t, z)\right] .
\end{array}
$$

These equations describe the effective coupling between the NF modes arising due to the deviations from the pure NLSE, see also (Kazakopoulos and Moustakas, 2016)

Within the NFT-based transmission concept, we can use both continuous and discrete spectra as data carriers (Le et al., 2017). In this work we we generate a pulse in a frequency domain, then apply the INFT to find the respective wave-shape in the time domain. After that we add the solitonic nonlinear spectral component corresponding to the eigenvalue $\zeta_{\text {sol }}$ 
using the Darboux transform (Aref et al., 2018). The overall signal modulation and wave-from generation method is identical to that from (Le et al., 2017). After the modulation and synthesis stage the signal is launched into the optical fibre. At the receiver, located at the distance $z=L$, one performs the forward NFT operation to recover the spectral profile $r(\xi, L)$, and removes the accumulated phase rotation:

$$
r(\xi, L)=e^{4 i \xi^{2} L} \frac{\left(\xi-\zeta_{\text {sol }}\right)}{\left(\xi-\bar{\zeta}_{\text {sol }}\right)} r(\xi, z=0) .
$$

For the detailed scheme of the nonlinear inverse synthesis NFT transmission method see (Derevyanko et al., 2016) and for the details of the Darboux transform computation see (Aref, 2016; Aref et al., 2018). The general flowchart referring to the NFT-based transmission is given in Fig. 1.

The presence of ASE in the optical fibre translates into the effective noise inside the NF domain which can be presented as an effective additive terms $N(\cdot)$ in the compensated NF spectrum:

$$
\begin{gathered}
e^{-4 i \xi^{2} L} r(\xi, L)=r(\xi, 0)+N(\xi, r(\xi, 0)), \\
\zeta_{s o l}(L)=\zeta_{\text {sol }}(z=0)+N_{\text {sol }}\left(\zeta_{\text {sol }}, r(\xi, 0)\right) .
\end{gathered}
$$

This effective noise terms in the NF domain, $N(\xi, r(\xi, 0))$ and $N_{\text {sol }}\left(\zeta_{\text {sol }}, r(\xi, 0)\right)$, are inputdependent. The nosie $N$ describing the action on the discrete spectrum is not circularly polarised even in the absence of the solitary eigenvalues. The latter means that when identifying this noise we need to consider not only the covariance function $\mathbb{E}\left[N(\xi) \bar{N}\left(\xi^{\prime}\right)\right]$, but also the pseudocovariance $\mathbb{E}\left[N(\xi) N\left(\xi^{\prime}\right)\right]$. In (Derevyanko et al., 2016) the analytical expressions for both covariance and pseudocovariance of the NF domain noise were obtained, under assumption of ideal distributed amplification, high signal-to-noise ratio and sufficiently long distances. The resulting expressions have the form:

$$
\begin{gathered}
\mathbb{E}\left[N(\xi) N^{*}\left(\xi^{\prime}\right)\right]=2 D L \pi \delta\left(\xi-\xi^{\prime}\right) E_{1}(\xi), \\
\mathbb{E}\left[N(\xi) N\left(\xi^{\prime}\right)\right]=2 D L \pi \delta\left(\xi-\xi^{\prime}\right) E_{2}(\xi),
\end{gathered}
$$

where each $E_{i}(\xi)$ also depends on the effective power inside the NF domain since the noise is inputdependent. The explicit expressions for $E_{1}$ and $E_{2}$ in the absence od the discrete spectrum were approximately derived in the Gaussian approximation as follows (Derevyanko et al., 2016):

$$
\begin{gathered}
E_{1}(\xi)=1+|r(\xi, 0)|^{2}+|r(\xi, 0)|^{4}, \\
E_{2}(\xi)=r(\xi, 0)^{2} .
\end{gathered}
$$

Note that a more accurate theory for the discrete NF spectra perturbations resulted in the non-Gaussian statistics (Derevyanko et al., 2003; Shevchenko et al., 2015), so expressions (14), (15) are approximate and refer to the leading order properties. In addition to these two characteristics of the continuous NF spectra considered in the absence of discrete eigenvalues in our previous studies (Pankratova et al., 2018; Pankratova et al., 2018), in this work we also address the following quantity referring to the correlations between the continuous and discrete NF spectra parts:

$$
\mathbb{E}\left[N(\xi) N_{\text {sol }}\right]=2 D L E_{\text {sol }}\left(\xi, \zeta_{\text {sol }}\right) .
$$

The linear limits of the covariance and pseudocovariance, Eqs. (14), (15), correspond to the correlators of the linear system defined in the conventional Fourier domain with the appropriate replacement of the frequency variable $\xi$, because in this limit the NFT converges to the conventional Fourier transform. The latter occurs when one deals with the small effective power corresponding to the profile $r(\xi)$, i.e. when the magnitude of the carriers' coefficients $c_{k}$ in Eq. (17) tends to zero. Note also that, generally, the pseudodensity $E_{2}(\xi)$ is a complex-valued function.

In this work we use the sinc-based spectral waveform of subcarriers defined on the continuous nonlinear spectrum part, corresponding to the NFT implementation of OFDM modulation (Le et al., 2014; Le et al., 2015; Kazakopoulos and Moustakas, 2016; Le et al., 2017; Aref et al., 2018). The nonlinear spectrum has the following form:

$$
r(\xi, z=0)=\sum_{k=-N_{\mathrm{ch}} / 2}^{N_{\mathrm{ch}} / 2} c_{k} \operatorname{sinc}(2 \xi-k),
$$

where $c_{k}$ are the information-bearing complex coefficients that are randomly taken from a QPSK modulation constellation; $N_{c h}$ is the number of subcarriers, and $\operatorname{sinc}(x)=\sin (\pi x) /(\pi x)$.

In the current study, we numerically examine the properties of effective nonlinear noise arising inside the NF domain due to the progenitor ASE $\eta$, Eq. (1), affecting the signal evolution in the space-time domain. We note that the noise properties inside the $\mathrm{NF}$ domain have attracted a considerable attention recently due to the studies referring to the capacity estimates for the NFT-based optical transmission methods (Derevyanko et al., 2016; Shevchenko et al., 2015; Shevchenko et al., 2018; Tavakkolnia and Safari, 2017; Tavakkolnia et al., 2018), and also in view of the serious noise effect on the NFT-based transmission properties (Civelli et al., 2017).

Recently, we showed in (Pankratova et al., 2018; Pankratova et al., 2018) that the correlation properties of NFT noise depend on the signal power and propagation distance in a non-trivial way, deviating from the simplified theory presented in (Derevyanko et al., 


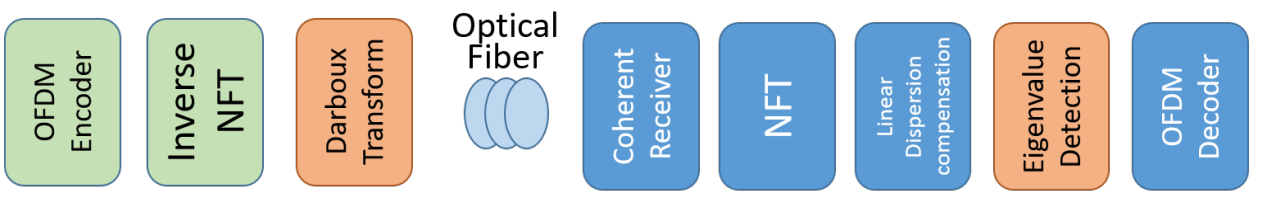

Figure 1: Basic elements of the communication system based on the nonlinear inverse synthesis with both continuous and discrete spectra present.

2016). In this paper we intend to consider the impact of discrete spectra on the correlation properties of the effective noise inside the NF domain and compare those with the case where the discrete eigenvalues are absent.

\section{Simulations and Results}

We consider the transmission of the optical signal down the single-mode fibre with standard parameters (Derevyanko et al., 2016; Aref et al., 2018) for the range of fiber lengths (up to $1500 \mathrm{~km}$ ) and $N_{\text {ch }}=128$ subcarriers. To investigate the properties of the effective noise inside the NF domain, we use the parameter $S=\left|c_{k}\right|^{2}$ as a measure effective signal power. In the QPSK modulation scheme involving only the phases of coefficients all symbols have the same power for the same absolute value of $c_{k}$. In Figs. 2 and 3, we present the results for the absolute values of the nonlinear power spectral density $\left|E_{1}(\xi)\right|(12)$ and an absolute value of the pseudodensity $\left|E_{2}(\xi)\right|(16)$, both versus the nonlinear frequency $\xi$, extracted from the numerical simulations for a particular realisation of randomly-modulated coefficients $c_{k}$ from (17), compared with the analytical expressions, Eqs. (11)-(16). In both figures the panes (a)-(d) represent the spectral density and pseudodencsity in the presence of a specific eigenvalue chosen from the set $\lambda_{1-4}=[1+i, 2+i, 1+2 i, 2+2 i]$.

Then we perform a comparison of the numerically obtained covariance density $E_{1}(\xi)$, Fig. 2, and pseudo-density $E_{2}(\xi)$, Fig. 3, with the analytical expressions using the propagation distance $500 \mathrm{~km}$. The black vertical line is marking the position of eigenvalue. We confirm that, as it was pointed out in (Pankratova et al., 2018; Pankratova et al., 2018), the theory tends to underestimate the true density of the NFT noise intensity. In the case considered here, i.e. when we have non-zero discrete spectrum, this observation can be explained by the fact that the analytical expression was derived for the continuous spectra only and in the assumption of large propagation distances and high SNR. In addition, it was shown in our previous works that covariance and pseudocavariance depend on the propagation distance, the fact which is
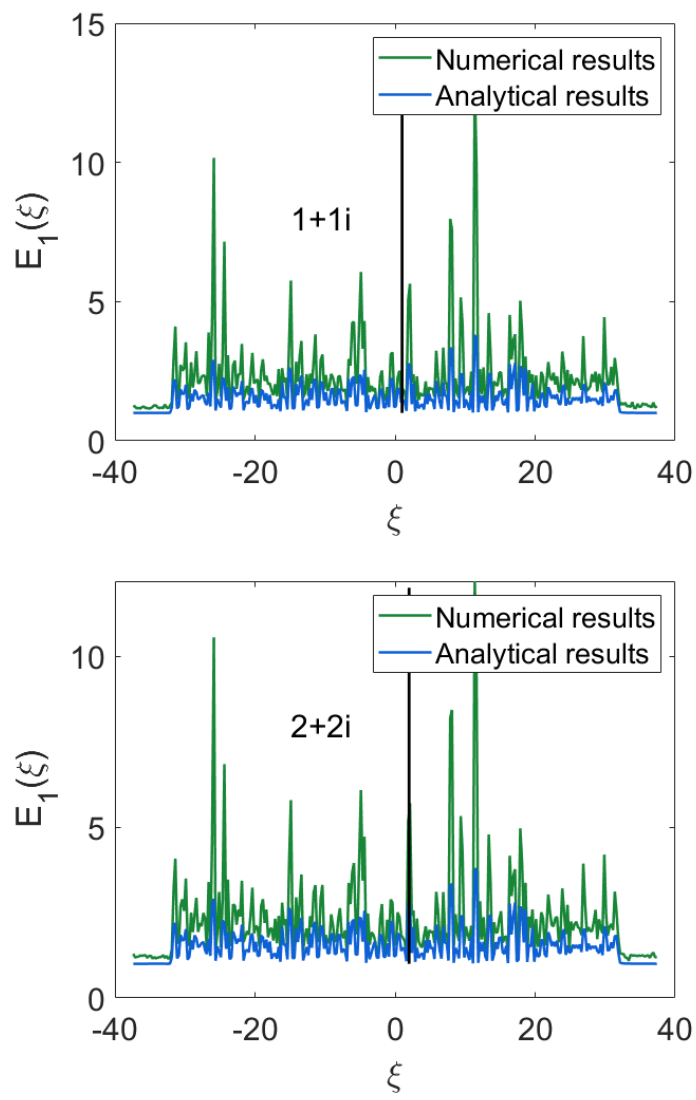

Figure 2: Power spectral density $\left|E_{1}(\xi)\right|$, Eq. (12) on the nonlinear frequency $\xi$, for a range of eigenvalue values from top to bottom $\lambda_{1,4}$ and for $L=500 \mathrm{~km}$. The effective power in the continuous NF spectrum is $S=0.405$.

not actually taken into account in the theory. Also, the numerical error emerging in the computation of NFT at the transmitter and receiver can result in the additional effective numerical noise.

In Fig. 4 we present the measure of the correlation between the continuous and discrete NF spectra, Eq. (16). It can be seen from the graph that there is a maximum related to the real part of the respective eigenvalue (marked by a straight vertical line) on each dependence. However, we also noticed the appearance of some additional maxima at other values of spectral parameter $\xi$ that require further studies.

In Fig. 5 we present the deviation of our nu- 

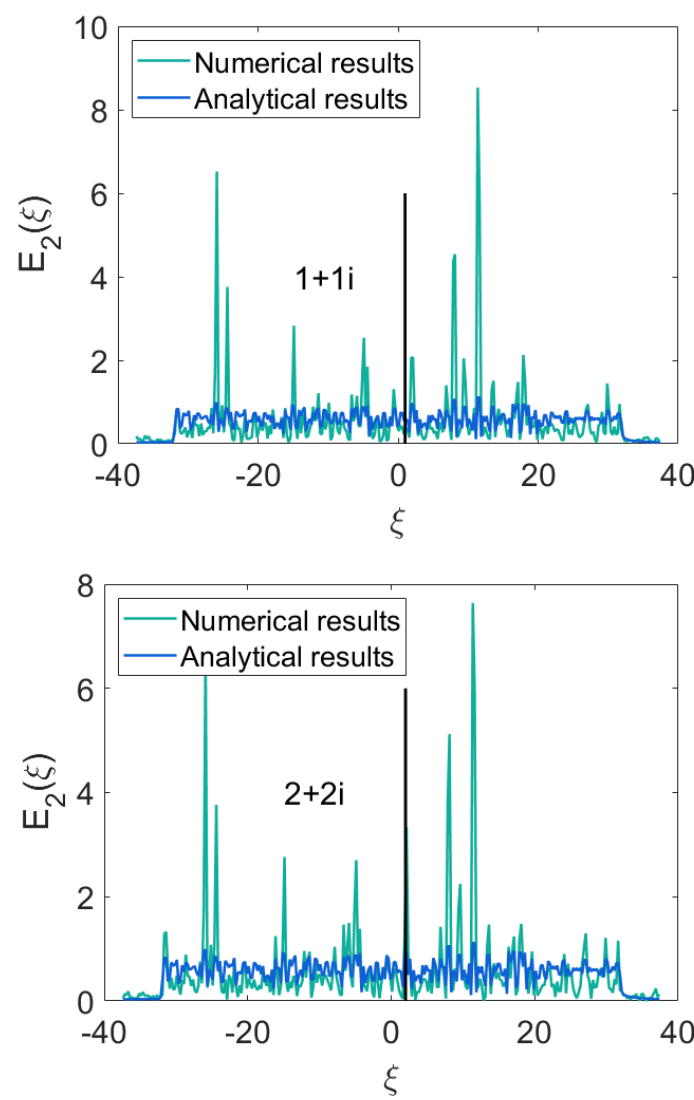

Figure 3: Power spectral pseudodensity $\left|E_{2}(\xi)\right|$, Eq. (16) on the nonlinear frequency $\xi$, for a range of eigenvalue values from top to bottom $\lambda_{1,4}$ and for $L=500 \mathrm{~km}$. The effective power in the continuous NF spectrum is $S=0.405$.

merical results from ones obtained analytically in (Derevyanko et al., 2016) as a function of distance for one value of signal power defined inside the NF domain, $S=0.45$. We present the relative deviation between our numerical calculation and analytical results in dependence on the propagation distance:

$$
\text { deviation }=\int\left|E_{1,2}^{\text {numerical }}(\xi)-E_{1,2}^{\text {analytical }}(\xi)\right| d \xi .
$$

It can be seen from that figure that the deviation is increasing with distance, since the theory is not taking into account the noise dependence on propagation distance. Also, additional simulations confirmed the observation from our previous works that the deviation is increasing with the growth of signal power.

\section{Conclusions}

In this work, the properties of the effective noise emerging in the NFT domain due to presence of pro-
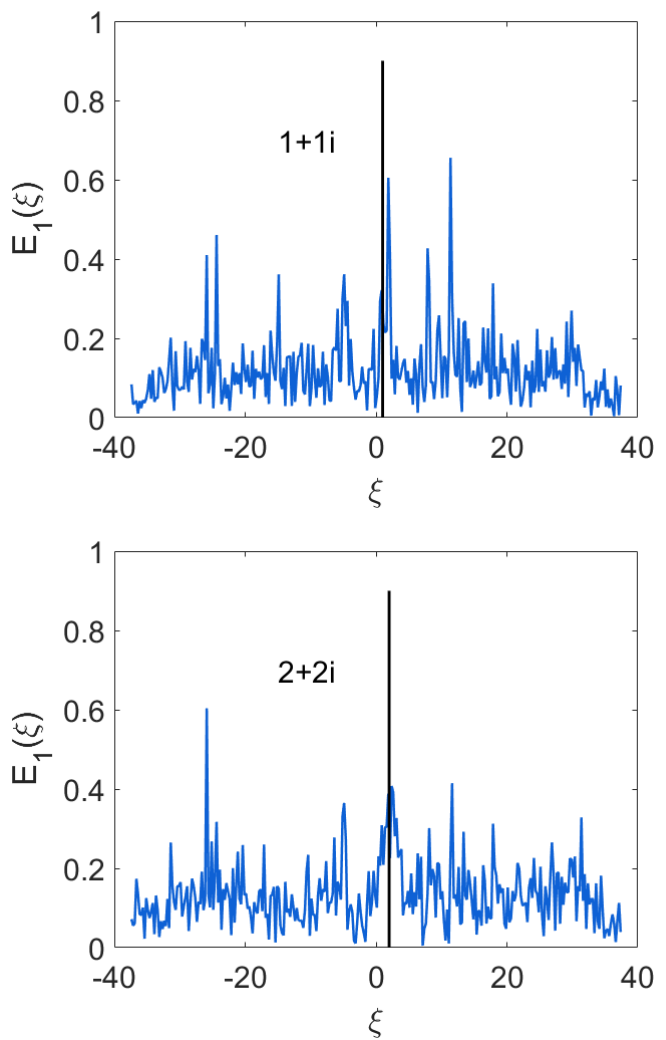

Figure 4: Correlation of continuous and discrete spectra dependence on the nonlinear frequency $\xi$, for a range of eigenvalue values from top to bottom $\lambda_{1,4}$ and for $L=500$ $\mathrm{km}$. The effective power in the continuous NF spectrum is $S=0.405$.

genitor ASE noise in the optical domain were investigated. In our study we took into account the presence of both continuous and discrete NF spectra parts. It is shown that the presence of eigenvalue affect continuous spectra in a non-trivial way: in addition to the peak referring to the real part of the eigenvalue we observed the appearance of some additional peaks at other values of parameter $\xi$. Our results have indicated that the channel defined inside the NF domain is a complicated nonlinear channel with the memory depending on the propagation distance, power, and on the presence of eigenvalues. Our findings can be used further to optimise the performance of NFTbased transmission systems with modulation of continuous and discrete spectra.

\section{Acknowledgements}

MLP: This project was supported by the Horizon 2020 programme under the MSCA-IF-EF-ST 


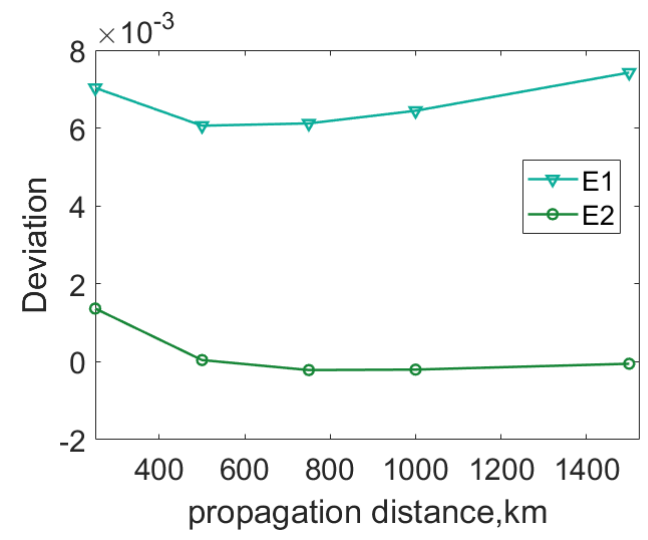

Figure 5: Deviation of our numerical results for covariance $E_{1}(\xi)$ and pseudocovariance $E_{2}(\xi)$ from theory proposed in (Derevyanko et al., 2016) as a function of propagation distance for $S=0.405$.

No 751561. JEP acknowledges the support of Leverhulme Project RPG-2018-063. JEP is thankful to Erasmus+ mobility programme. The authors acknowledge helpful comments of Dr S. A. Derevyanko.

\section{REFERENCES}

Agrawal, G. P. (2012). Fiber-optic communication systems, volume 222. John Wiley \& Sons.

Aref, V. (2016). Control and detection of discrete spectral amplitudes in nonlinear fourier transform. Arxiv.org.

Aref, V., Le, S., and Buelow, H. (2018). Modulation over nonlinear fourier spectrum: Continuous and discrete spectrum. Journal Lightwave Technologies, 36:1289.

Civelli, S., Forestieri, E., and Secondini, M. (2017). Why noise and dispersion may seriously hamper nonlinear frequency-division multiplexing. IEEE Photonics Technology Letters, 29(16):1332-1335.

Derevyanko, S. A., Prilepsky, J. E., and Turitsyn, S. K. (2016). Capacity estimates for optical transmission based on the nonlinear fourier transform. Nature Commun, 7(12710):307.

Derevyanko, S. A., Turitsyn, S., and Yakushev, D. (2003). Non-gaussian statistics of an optical soliton in the presence of amplified spontaneous emission. Optics letters, 28(21):2097-2099.

Essiambre, R.-J., Kramer, G., Winzer, P. J., Foschini, G. J., and Goebel, B. (2010). Capacity limits of optical fiber networks. Journal of Lightwave Technology, 28(4):662-701.

Garcia-Gomez, F. J. and Aref, V. (2019). Statistics of the nonlinear discrete spectrum of a noisy pulse. arXiv preprint arXiv:1901.11419.

Kamalian, M., Prilepsky, J. E., Le, S. T., and Turitsyn, S. K. (2017). On the design of nft-based communication systems with lumped amplification. Journal of Lightwave Technology, 35(24):5464-5472.

Kaup, D. (1976). A perturbation expansion for the zakharov-shabat inverse scattering transform. SIAM Journal on Applied Mathematics, 31(1):121-133.

Kaup, D. J. and Newell, A. C. (1978). Solitons as particles, oscillators, and in slowly changing media: a singular perturbation theory. Proceedings of the Royal Society of London. A. Mathematical and Physical Sciences, 361(1707):413-446.

Kazakopoulos, P. and Moustakas, A. L. (2016). On the soliton spectral efficiency in non-linear optical fibers. In 2016 IEEE International Symposium on Information Theory (ISIT), pages 610-614. IEEE.

Le, S., Aref, V., and Buelow, H. (2017). Nonlinear signal multiplexing for communication beyond the kerr nonlinearity limit. Nature Photonics, 11:570.

Le, S. T., Prilepsky, J. E., and Turitsyn, S. K. (2014). Nonlinear inverse synthesis for high spectral efficiency transmission in optical fibers. Optics Express, 22:26270.

Le, S. T., Prilepsky, J. E., and Turitsyn, S. K. (2015). Nonlinear inverse synthesis technique for optical links with lumped amplification. Optics express, 23(7):8317-8328.

Pankratova, M., Vasylchenkova, A., Prilepsky, J. E., and Derevyanko, S. A. (2018). Properties of the effective noise in the nonlinear fourier transform-based transmission. In Frontiers in Optics, pages JW3A-83. Optical Society of America.

Pankratova, M. L., Vasylchenkova, A., Prilepsky, J., and Derevyanko, S. A. (2018). Study of noise-induced signal corruption for nonlinear fourier-based optical transmission. In 2018 IEEE British and Irish Conference on Optics and Photonics (BICOP), pages 1-3.

Shevchenko, N. A., Derevyanko, S. A., Prilepsky, J. E., Alvarado, A., Bayvel, P., and Turitsyn, S. K. (2018). Capacity lower bounds of the noncentral chi-channel with applications to soliton amplitude modulation. IEEE Transactions on Communications, 66(7):29782993.

Shevchenko, N. A., Prilepsky, J. E., Derevyanko, S. A., Alvarado, A., Bayvel, P., and Turitsyn, S. K. (2015). A lower bound on the per soliton capacity of the nonlinear optical fibre channel. In 2015 IEEE Information Theory Workshop-Fall (ITW), pages 104-108. IEEE.

Tavakkolnia, I., Alvarado, A., and Safari, M. (2018). Capacity estimates of single soliton communication. In 2018 European Conference on Optical Communication $(E C O C)$, pages 1-3. IEEE.

Tavakkolnia, I. and Safari, M. (2017). Capacity analysis of signaling on the continuous spectrum of nonlinear optical fibers. Journal of Lightwave Technology, 35(11):2086-2097.

Turitsyn, S. K., Prilepsky, J. E., Le, S. T., Wahls, S., Frumin, L. L., Kamalian, M., and Derevyanko, S. A. (2017). Nonlinear fourier transform for optical data processing and transmission: advances and perspectives. $O p$ tica, 4:307. 
Vasylchenkova, A., Prilepsky, J., Shepelsky, D., and Chattopadhyay, A. (2019). Direct nonlinear fourier transform algorithms for the computation of solitonic spectra in focusing nonlinear schrödinger equation. Communications in Nonlinear Science and Numerical Simulation, 68:347-371.

Yousefi, M. and Kschischang, F. (2014). Information transmission using the nonlinear fourier transform, part iiii. IEEE Trans. Inf. Theory, 60:4312.

Zakharov, V. E. and Shabat, A. B. (1972). Exact theory of 2-dimensional self-focusing and one-dimensional self-modulation of waves in nonlinear media. Soviet Physics-JETP, 34:62. 